THE AsTROPHYSICAL JOURNAL, 535:928-931, 2000 June 1

(c) 2000. The American Astronomical Society. All rights reserved. Printed in U.S.A.

\title{
MEASURING THE REMNANT MASS FUNCTION OF THE GALACTIC BULGE
}

\author{
ANDREW GOULD \\ Department of Astronomy, Ohio State University, Columbus, OH 43210; gould@astronomy.ohio-state.edu \\ Received 1999 June 29; accepted 2000 January 18
}

\begin{abstract}
I show that by observing microlensing events both astrometrically and photometrically, the Space Interferometry Mission (SIM) can measure the mass function of stellar remnants in the Galactic bulge, including white dwarfs, neutron stars, and black holes. Neutron stars and black holes can be identified individually, while white dwarfs are detected statistically from the sharp peak in their mass function near $M \sim 0.6 M_{\odot}$. This peak is expected to be more than twice as high as the "background" of mainsequence microlenses. I estimate that of order $20 \%$ of the $\sim 400$ bulge microlensing events detected to date are due to remnants, but show that these are completely unrecognizable from their timescale distribution (the only observable that "normal" microlensing observations produce). To resolve the white dwarf peak, the SIM mass measurements must be accurate to $\sim 5 \%$, substantially better than is required to measure the mass function of the more smoothly distributed main sequence. Nevertheless, SIM could measure the masses of about 20 bulge remnants in 500 hours of observing time.
\end{abstract}

Subject headings: astrometry - black hole physics - gravitational lensing - stars: neutron white dwarfs

\section{INTRODUCTION}

There are two reasons that one would like to measure the mass function (MF) of stellar remnants in the Galactic bulge, including white dwarfs (WDs), neutron stars (NSs), and black holes (BHs). First, stellar remnants are one of only two fossils of the era of high-mass star formation in the bulge (the other being metal abundances). They can therefore help determine the stellar MF for masses $M \gtrsim M_{\odot}$, a regime that is otherwise virtually inaccessible in the bulge.

Second, a fair sample of remnants in any environment is difficult to obtain, so measuring the MF of bulge remnants would shed light on the study of remnants in clusters, in the field, and perhaps in other systems as well. For example, NSs can generally be detected only if they are pulsars, and it is difficult to estimate what fraction of all NSs are currently detectable pulsars. Moreover, the masses of NSs can only be measured if these are found in suitable binary systems. While the range of masses found by this technique is impressively narrow, $M_{\mathrm{NS}}=1.35 \pm 0.04 M_{\odot}$ (Thorsett \& Chakrabarty 1999), this could in principle be due to the narrow range of systems that have been investigated. By contrast, WDs can be located in systematic searches of the solar neighborhood. However, the WD MF derived from the subsamples with measurable masses is controversial. For example, based on a sample of 164 hot (and so recently formed) WDs, Bragaglia, Renzini, \& Bergeron (1995) find a WD MF that is sharply peaked at $M \sim 0.6 M_{\odot}$ with a dispersion (excluding four He core WDs) of $0.16 M_{\odot}$ that is mainly generated by a long tail toward high masses. On the other hand, Silvestri et al. (1999) find a substantially higher dispersion of $0.25 M_{\odot}$ from a sample of 70 cooler, old WDs found in common proper-motion binaries. They argue that their sample is more representative of the population as a whole. To date, BHs have been found only when they are in relatively close binaries, and even these are quite difficult to confirm or to measure their masses (as opposed to obtaining a lower limit). The frequency of field BHs is virtually unconstrained.

The problem of obtaining a remnant MF for the bulge appears formidable. A few WDs are bright enough to detect, but typical bulge WDs are $V \sim 30$. Serious effort would be required merely to detect such objects in a high-latitude field, let alone measure their mass. In crowded bulge fields, optical detection is virtually impossible. NSs and BHs are even more difficult to detect.

However, it is straightforward to detect all three classes of objects, using gravitational microlensing. Indeed, I will argue below that of order 80 of $\sim 400$ microlensing events seen toward the bulge to date (Udalski et al. 1994b; Alcock et al. 1997) are due to bulge remnant lenses. The only problem is that no one has the least idea which 80 they are.

Here I discuss how observations using the Space Interferometry Mission (SIM) can measure important features of the bulge remnant MF. First, I show that from their timescales alone (normally the only useful parameter extracted from a microlensing event), the remnants cannot be separated from the main-sequence (MS) stars, even statistically. However, Gould \& Salim (1999) have shown that if SIM astrometry is combined with photometry from both the ground and from SIM itself, then SIM can measure of order 100 masses of microlensing events to $\sim 5 \%$ precision in about 500 hours of observation. This sample should contain of order $15 \mathrm{WDs}$ and five higher mass remnants, NSs and BHs. Such a measurement would be adequate to pick out the peak of the WD MF and make a rough determination of the frequency of NSs and BHs. To make a more detailed measurement of the bulge remnant MF would require a mission similar to SIM but with a substantially larger aperture.

\section{MAIN-SEQUENCE VERSUS REMNANT STARS}

The MF of the bulge MS has been measured in both the optical (Holtzman et al. 1998) and the infrared (Zoccali et al. 2000) using Hubble Space Telescope (HST) observations. For purposes of this paper, I will adopt a MS MF that is consistent with those measurements (but without corrections for binaries),

$$
\frac{d N}{d M}=k\left(\frac{M}{M_{\mathrm{brk}}}\right)^{\alpha}, \quad M_{\mathrm{brk}}=0.7 M_{\odot},
$$


where $k$ is a constant, and

$$
\begin{gathered}
\alpha=-1.3 \quad\left(0.15 M_{\odot}<M<M_{\text {brk }}\right), \\
\alpha=-2.0 \quad\left(M_{\text {brk }}<M \lesssim M_{\odot}\right) .
\end{gathered}
$$

The upper limit of $\sim 1 M_{\odot}$ is the approximate position of the turnoff. The lower limit of $0.15 M_{\odot}$ comes from the magnitude limit of the Zoccali et al. (2000) observations. The MF probably continues below this limit, but at present it is not known how far. The change in slope in the true MF is perhaps less abrupt than is represented in equation (1). The MF should also be corrected for binaries. In the Appendix, I discuss how this correction can be observationally determined. I will assume that the slope of $\alpha=-1.3$ observed to $M \sim 0.15$ actually extends to $M=$ $0.03 M_{\odot}$,

$$
\alpha=-1.3 \quad\left(0.03 M_{\odot}<M<M_{\text {brk }}\right) .
$$

It may well extend further, but SIM will probably not be sensitive to much lower masses because the events are too short to be alerted in time for SIM observations. Thus, equations (1)-(3) are a rather approximate representation of the bulge MS MF. However, I will mainly be using the MS MF for illustration, and for that purpose this approximation is quite adequate.

I will assume that all MS stars in the range $1 M_{\odot}<$ $M<8 M_{\odot}$ have now become WDs, and that the total number can be found by extending the upper MS power law $\alpha=-2.0$ through this higher mass regime. That is, $N_{\mathrm{wD}}=$ $\frac{7}{8} k M_{\text {brk }}$. Of course, there is no evidence whatever that the slope does continue in this regime. A more popular slope is the Salpeter value $\alpha=-2.35$. Had I chosen this steeper slope, my estimate for $N_{\text {WD }}$ would be reduced by a factor 0.80 . For the distribution of WD masses, I adopt the MF shown in Figure 11c of Bragaglia et al. (1995) based on observations of 164 hot WDs. I assume that all MS stars with $8 M_{\odot}<M<40 M_{\odot}$ become NSs, with masses that are centered at $M=1.35 M_{\odot}$ and with Gaussian dispersion of $0.04 M_{\odot}$ (Thorsett \& Chakrabarty 1999). I assume that all MS stars with $40 M_{\odot}<M<100 M_{\odot}$ become BHs, with masses that are centered at $M=5 M_{\odot}$ and with Gaussian dispersion $1 M_{\odot}$. I have no justification for this BH distribution, but since BHs with $M \gtrsim 2 M_{\odot}$ will be easily identified by SIM whatever their mass (see $\S 3$ ), the particular form of their distribution can be fixed arbitrarily. I also assume that the power law $\alpha=-2$ extends throughout this entire regime. Again, this assumption is arbitrary, but it is appropriate for purposes of illustration.

With these assumptions, the fractions of numbers $N$ of objects in the four classes, MS stars (including brown dwarfs, $\left.M>0.03 \quad M_{\odot}\right)$, WDs, NSs, and BHs are $(89: 10: 1: 0.2)$. The fractions of the total mass (which scales $\propto N M)$ are $(69: 22: 6: 3)$. The fractions of microlensing events (which scale $\propto N M^{1 / 2}$ ) are $(79: 17: 3: 0.8)$. That is, of order $20 \%$ of the bulge microlensing events are due to remnants.

\subsection{Combined Mass Function}

Figure $1 a$ shows the distribution of microlensing events per unit log mass, as a function of log mass and classified by type of object. It is normalized to a total of 100 bulge microlensing events. Gould \& Salim (1999) showed that masses of approximately 100 microlensing events could be measured by SIM. Of these, only $\sim 80 \%$ should be due to bulge (rather than disk) lenses. Nevertheless, for simplicity I nor- malize to 100 events. The WD bins in Figure $1 a$ are 0.05 $M_{\odot}$ wide. Notice that the peak WD bin (which contains about one-third of all the WDs) stands well above the "background" of MS stars, and that there are several adjacent WD bins that are comparable to the MS background. If 100 individual masses were measured, then about six stars would be in this peak bin and another six would be in the two neighboring bins toward higher mass. From these three bins alone, I find a total signal-to-noise ratio, $\mathrm{S} / \mathrm{N}=$ $\left[\sum_{i} N_{\mathrm{WD}, i}^{2} /\left(N_{\mathrm{WD}, i}+N_{\mathrm{MS}, i}\right)\right]^{1 / 2} \sim 3$, where $N_{\mathrm{WD}, i}$ and $N_{\mathrm{MS}, i}$ are the numbers of WD and MS stars in bin $i$. This implies a statistical accuracy in the WD frequency of about $33 \%$. The NSs and BHs would be free of any MS background, so their frequency measurement would be limited by Poisson errors, roughly $50 \%$ for a combined total of four stars.

\subsection{Timescale Distribution at Fixed Mass}

I have previously shown that if one assumes that the bulge lenses are distributed as $r^{-2}$, that the lenses and sources each have Gaussian velocity distributions with onedimensional dispersion $\sigma$, and that all the sources are at the Galactocentric distance, then the timescale distribution for lenses at fixed mass $M$ is given by

$$
\frac{d \Gamma}{d \ln t_{\mathrm{E}}} \propto t_{\mathrm{E}}^{-1} \int_{0}^{\infty} d z \frac{z^{2}}{z^{2}+\left(t_{b M} / t_{\mathrm{E}}\right)^{4}} \exp (-z)
$$

where $t_{\mathrm{E}}$ is the Einstein crossing time,

$$
t_{b M} \equiv \frac{(G M b)^{1 / 2}}{\sigma c},
$$

and $b$ is the impact parameter of the line of sight relative to the Galactic center (Gould 1995b). My excuse for assuming that all the sources were at the Galactocentric distance was that it made the problem " analytically tractable," but probably I just forgot to put on my thinking cap: If all the sources were assumed to be at the Galactocentric distance, then the distribution of source-lens separations, $d_{1 \mathrm{~s}}$ (not weighted by the lensing cross sections), would be just $f_{0}\left(d_{1 \mathrm{~s}} ; b\right)=K\left(b^{2}+d_{1 \mathrm{~s}}^{2}\right)^{-1}$, where $K$ is a constant. If we now assume that the lenses and sources are both distributed as $r^{-2}$, then the distribution of separations is

$$
\begin{aligned}
f\left(d_{\mathrm{ls}} ; b\right) & =\frac{\int d x d y f_{0}(x ; b) f_{0}(y ; b) \delta\left(y-x-d_{\mathrm{ls}}\right)}{\int d x f_{0}(x ; b)} \\
& =\frac{2 K}{d_{\mathrm{ls}}^{2}+4 b^{2}}=2 f_{0}\left(d_{\mathrm{ls}} ; 2 b\right),
\end{aligned}
$$

where $\delta$ is a Dirac $\delta$-function. That is, equation (4) remains valid but with

$$
t_{b M} \rightarrow \frac{(2 G M b)^{1 / 2}}{\sigma c} .
$$

Figure $1 b$ shows the distribution of events per log timescale as a function of $\log \left(t_{\mathrm{E}} / t_{b M}\right)^{2}$. The reason for using this scale (which of course is simply double the natural scale of $\left.\log \left(t_{\mathrm{E}} / t_{b M}\right)\right)$ is that for microlensing events, $t_{\mathrm{E}}^{2} \propto M$. Thus Figures $1 a$ and $1 b$ can be directly compared. This comparison reveals that the width of the timescale distribution at fixed mass is enormously larger than the scale on which the remnant populations have structure and is therefore likely to obliterate any distinctive traces of the remnants.

Figure $1 c$ demonstrates that this is indeed the case. It shows the distribution of timescales that will be observed 

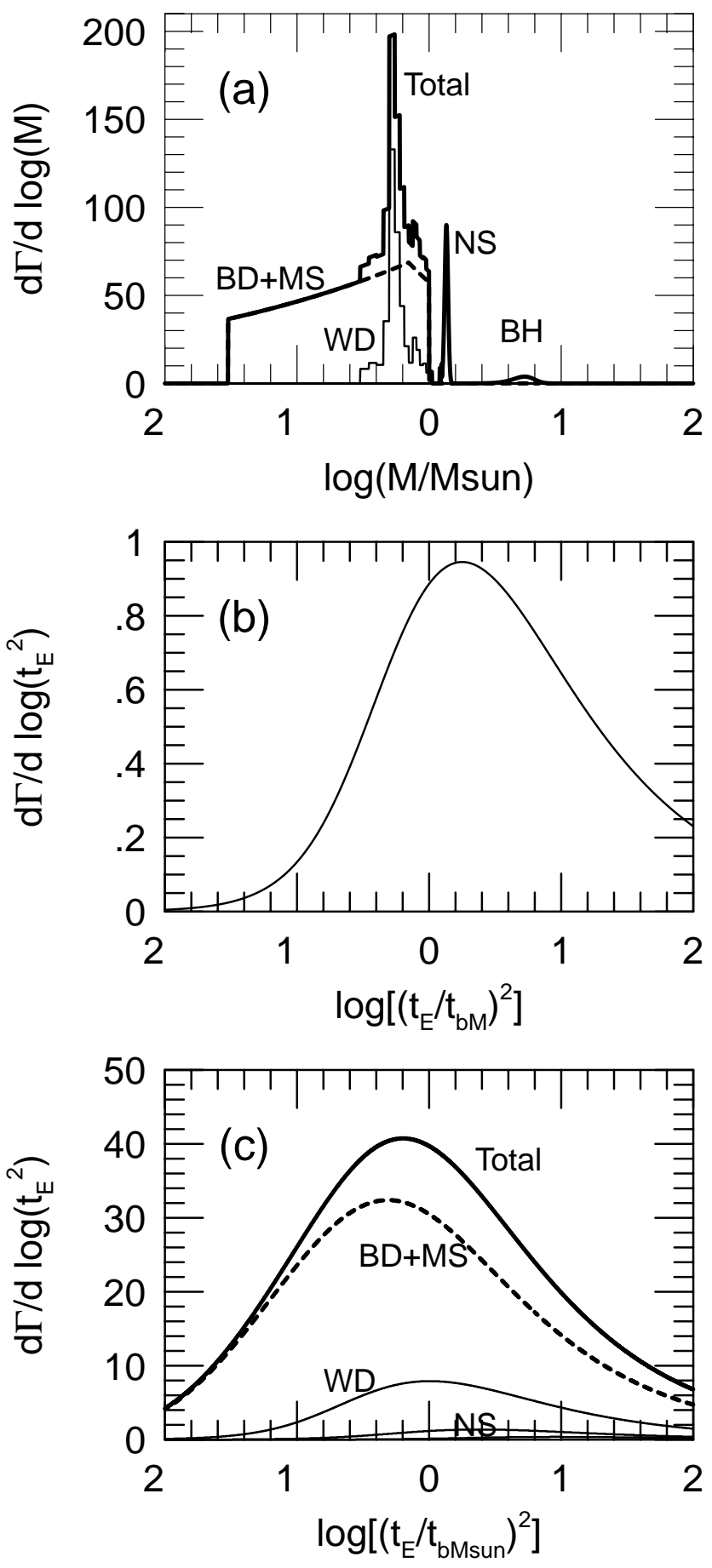

FIG. 1.-Rates of microlensing events toward the bulge by mass $(a)$ and timescale $(c)$ for MS stars and brown dwarfs $\left(0.03 M_{\odot}<M<1 M_{\odot}\right)$ (bold dashed curve) and WD, NS, and BH remnants (solid curves). The total is shown by a bold solid curve. The mass model $(a)$ is described in $\S 2$. In particular, the WD distribution is shown in $0.05 M_{\odot}$ bins taken from Bragaglia et al. (1995). The mass model is convolved with the timescale distribution at fixed mass $(b)$ derived in $\S 2.2$, to produce the observable timescale distribution $(c)$. The abscissae of $(b)$ and $(c)$ contain $\log t_{\mathrm{E}}^{2}$ rather than $\log t_{\mathrm{E}}$ so that they can be directly compared with $(a)$, since $t_{\mathrm{E}}^{2} \propto M$. All three classes of remnants are clearly identifiable in the mass distribution but are utterly lost in the timescale distribution. The normalizations in $(a)$ and $(c)$ are for 100 events; $(b)$ is normalized to unity.

from the mass distribution in Figure $1 a$ given the timescale distribution at fixed mass shown in Figure $1 b$. Figure $1 c$ is simply the convolution of Figures $1 a$ and $1 b$. The timescale normalization parameter is $t_{b M_{\odot}}=\left(2 G M_{\odot} b\right)^{1 / 2} / \sigma c$. The
WD peak is now so spread out that it cannot be distinguished from the MS. The NS and BH peaks are also spread out, but the main problem is that, in constrast to Figure $1 a$, they are now overwhelmed by background from the much more numerous MS stars whose timescale distribution is smeared out over the entire range illustrated in the figure. The conclusion is that, while a significant fraction of microlensing events are due to remnants, these remnants will never be recognized as such from their observed timescales alone.

\section{MEASURING THE REMNANT MASS FUNCTION WITH SIM}

For bright bulge sources $(I \sim 15)$ SIM can measure the masses of bulge lenses with $\sim 5 \%$ precision in about 5 hours of observing time (Gould \& Salim 1999). From Figure $1 a$ (where the WDs are binned by $0.05 M_{\odot}$ ), it is clear that approximately this precision is required to resolve the WD peak and so be able to efficiently separate the WDs from the MS background. Indeed, the mass errors reported by Bragaglia et al. (1995) when they constructed the WD MF used as a basis for Figure $1 a$ are of order $5 \%$. Thus, if the bulge WD MF is similar to the hot WD MF investigated by Bragaglia et al. (1995), then the distribution of masses measured by SIM (including 5\% errors) should look fairly similar to the one shown in Figure $1 a$.

Note that if one were concerned only to measure the mass function of main-sequence stars, the precision requirements could be relaxed significantly because the main sequence does not exhibit structure on small scales. In fact, for the main sequence the precision is required more to measure the lens-source relative parallax (which has the same fractional error as the mass; Gould \& Salim 1999) in order to be able to separate bulge lenses from disk lenses.

The measurement requirements for NSs and especially BHs are less severe than for WDs. NSs are $\sim 35 \%$ heavier than turnoff stars, so if their masses could be measured to $\sim 10 \%$, they could be reliably distinguished from MS stars. Moreover, within the model I am considering, events due to upper MS stars $\left(0.7 M_{\odot}<M<1.0 M_{\odot}\right)$ are only about 3 times as common as those due to NSs, so the tail of the MS event distribution will not seriously corrupt the measurement of the NS frequency.

Unfortunately, for fixed SIM observation time, the fractional error in the mass measurement grows with mass (Gould \& Salim 1999). The reason for this is that two quantities must be separately measured to determine $M$, the angular Einstein radius $\theta_{\mathrm{E}}$ (Boden, Shao, \& Van Buren 1998), and the size of the Einstein radius projected onto the plane of the observer, $\tilde{r}_{\mathrm{E}}$ (Gould 1995a). Specifically $M=$ $\left(c^{2} / 4 G\right) \tilde{r}_{\mathrm{E}} \theta_{\mathrm{E}}$. Both $\theta_{\mathrm{E}}$ and $\tilde{r}_{\mathrm{E}}$ scale $\propto M^{1 / 2}$. As $\theta_{\mathrm{E}}$ grows, the astrometric deviation grows with it and so becomes easier to measure. However, $\tilde{r}_{\mathrm{E}}$ is measured from the difference in the photometric light curves as seen from the Earth and $S I M$. The bigger $\tilde{r}_{\mathrm{E}}$, the closer the Earth and SIM are in the projected Einstein ring, and the harder it is to measure the difference in the light curves as seen from the two observatories. Thus, observations that would be sufficient to obtain $5 \%$ errors for "typical" stars $\left(M \sim 0.3 M_{\odot}\right)$ achieve only about $10 \%$ precision for NSs of $1.35 M_{\odot}$, and $30 \%$ precision for BHs of $5 M_{\odot}$. However, since BHs are separated from the MS by a factor of a few in mass, even errors of order $30 \%$ would be sufficient to recognize them as such.

Thus, the program outlined by Gould \& Salim (1999) 
would be adequate to recognize NSs and BHs individually whenever the events were observed, and would also suffice to recognize the peak of the WD mass function. The main limitation of this program is its modest statistics: fewer than $1 \mathrm{BH}$ event and only 3 NS events are expected.

In principle it would be possible to overcome this problem simply by observing more events. However, the observation time scales inversely as the source flux. There are a limited number of events with bright sources. If one wanted to increase the number of measurements by a factor of 2 and maintain the same errors, one would be forced to observe fainter sources and hence substantially increase the observing time per source. Thus, the observation time would grow much more rapidly than the number of measurements. It therefore appears that the only feasible route to fainter sources (and thus substantially more events) is a mission similar to $S I M$ but with apertures that are larger than the $25 \mathrm{~cm}$ mirrors on SIM.

I would like to thank S. Salim for calculating the SIM errors for NS and BH masses, and B. S. Gaudi for a careful reading of the manuscript. This research was supported in part by grant AST 97-27520 from the NSF and in part by grant NAG5-3111 from NASA.

\section{APPENDIX}

\section{HOW TO DETERMINE THE BULGE BINARY DISTRIBUTION}

The correction for binaries remains an important uncertainty in the mass budget of the bulge. Binaries in the bulge, in sharp contrast to those in the solar neighborhood (e.g., Duquennoy \& Mayor 1991) or even in globular clusters (Hut et al. 1992), are virtually unstudied. Here I briefly outline how they could be. Close binaries of roughly solar mass primaries could be studied from eclipsing binaries found in microlensing studies (Udalski et al. 1994a, 1995a, 1995b) and radial velocity measurement of clump giants. The probability that a binary is an eclipsing system declines inversely as the semimajor axis, and the fraction of the period spent in eclipse falls at the same rate, so this technique can probably be extended only to $\sim 100$ stellar radii, or about $1 \mathrm{AU}$ for turnoff sources. For substantially brighter sources the eclipses are too shallow to detect easily, and for substantially fainter sources the source is too faint to monitor with current or foreseeable programs. An extensive radial velocity survey requires bright sources of which the most numerous are clump giants. These can be searched for companions from $\sim 0.5 \mathrm{AU}$ (inside of which the companion may have been affected during the red giant phase) to $\sim 5 \mathrm{AU}$ (beyond which the orbital periods become too long to monitor).

Microlensing surveys can effectively search for binaries over the range $0.2 \lesssim b \lesssim 30$, where $b \equiv r_{p} / r_{\mathrm{E}}, r_{p}$ is the projected separation, and $r_{\mathrm{E}}$ is the Einstein radius, which for bulge lensing events is roughly given by $r_{\mathrm{E}} \sim 3.5\left(M / M_{\odot}\right)^{1 / 2}$ AU. For $b \lesssim 0.2$, the binary microlensing event behaves photometrically like a point mass (Gaudi \& Gould 1997) and so cannot be recognized (although binaries with even smaller separations can be recognized astrometrically; Chang \& Han 1999). For $0.2 \lesssim b \lesssim 2$ and for a significant fraction of events, the binary produces characteristic caustic structures that are easily recognized. For $2 \lesssim b \lesssim 30$, the binary gives rise to two-peaked events (Di Stefano \& Scalzo 1999). Although some of the automated routines used by microlensing search teams might throw these events out on the grounds that "microlensing events do not repeat," there are hundreds of events that have been recognized in real time ("alerts"), and these could be searched for second peaks up to several years after the first bump. The major limitation is that the probability of a second bump falls off as $b^{-1}$. In addition, one must wait $b$ Einstein crossing times (each typically $t_{\mathrm{E}} \sim 30\left(M / M_{\odot}\right)^{1 / 2}$ days) for the bump to occur, and this may exceed the duration of the microlensing experiment, which in general has a long, but finite, lifetime.

Finally, it should be possible to search for common proper-motion pairs by comparing two Hubble Space Telescope observations. For example, the first epoch could be taken from Holtzman et al. (1998). It should be possible to measure positions from a single image accurate to 2 mas (I. King 1999, private communication), and so with a 5 year baseline (and multiple exposures at each epoch) easily distinguish common proper-motion pairs from optical binaries whose typical

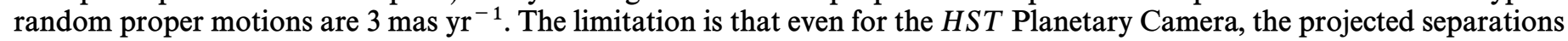
must be 0 "' 15 or more (see Table 1 of Gould et al. 1995) depending on the magnitude difference just to detect separate stars. This corresponds to $r_{p} \sim 1200 \mathrm{AU}$ at the Galactocentric distance.

By combining all these techniques, one could cover separations $r_{p} \lesssim 100 \mathrm{AU}$ and $r_{p} \gtrsim 1200 \mathrm{AU}$. Although a substantial range of separations would be inaccessible, one could determine whether the basic pattern found by Duquennoy \& Mayor (1991) for solar-type primaries in the solar neighborhood also holds true for the bulge. If it did, one could interpolate into the unobserved interval $100 \mathrm{AU} \lesssim r_{p} \lesssim 1200 \mathrm{AU}$ using the local data.

\section{REFERENCES}

Alcock, C., et al. 1997, ApJ, 479, 119

Boden, A. F., Shao, M., \& Van Buren, D. 1998, ApJ, 502, 538

Bragaglia, A., Renzini, A., \& Bergeron, P. 1995, ApJ, 443, 735

Chang, K., \& Han, C. 1999, ApJ, 525, 434

Di Stefano, R., \& Scalzo, R. A. 1999, ApJ, 512, 579

Duquennoy, A., \& Mayor, M. 1991, A\&A, 248, 485

Gaudi, B. S., \& Gould, A. 1997, ApJ, 482, 83

Gould, A. 1995a, ApJ, 441, L21 $1995 \mathrm{~b}$, ApJ, 446, L71

Gould, A., Bahcall, J. N., Maoz, D., \& Yanny, B. 1995, ApJ, 441, 200

Gould, A., \& Salim, S. 1999, ApJ, 524, 794

Holtzman, J. A., Watson, A. M., Baum, W. A., Grillmair, C. J., Groth, E. J.,

Light, R. M., Lynds, R., \& O’Neil, E. J., Jr. 1998, AJ, 115, 1946
Hut, P., et al. 1992, PASP, 104, 981

Silvestri, N. M., Oswalt, T. D., Wood, M. A., Smith, J. A., Reid, N., \& Sion, E. M. 1999, BAAS, 194, 52.03

Thorsett, S. E., \& Chakrabarty, D. 1999, ApJ, 512, 288

Udalski, A., Olech, A., Szymanski, M., Kaluzny, J., Kabuki, K, Mateo, Me., \& Krzeminski, W. 1995a, Acta Astron., 45, 433

Udalski, A., Szymanski, M., Kaluzny, J., Kubiak, K, Mateo, M., \& Krzeminski, W. 1994a, Acta Astron., 44, 317 1995b, Acta Astron., 45, 1

Udalski, A., et al. 1994b, Acta Astron., 44, 165

Zoccali, M. S., Cassisi, S., Frogel, J. A., Gould, A., Ortolani, S., Renzini, A., \& Rich, R. M., \& Stephens, A. 2000, ApJ, 530, 418 